\title{
Is the massive young cluster Westerlund I bound?
}

\author{
M. Cottaar ${ }^{1}$, M. R. Meyer ${ }^{1}$, M. Andersen ${ }^{2}$, and P. Espinoza ${ }^{3}$ \\ 1 Institute for Astronomy, ETH Zurich, Wolfgang-Pauli-Strasse 27, 8093 Zurich, Switzerland \\ e-mail: michielcottaar@gmail.com \\ 2 European Space Agency (ESTEC), PO Box 299, 2200 AG Noordwijk, The Netherlands \\ 3 Steward Observatory, The University of Arizona, Tucson, Arizona 85721, USA
}

Received 18 July 2011 / Accepted 12 December 2011

\section{ABSTRACT}

\begin{abstract}
Context. Westerlund I is the richest young cluster currently known in our Galaxy, making it one of the most massive clusters for which we can resolve the individual stars even in the crowded centre. This makes it an ideal target to assess whether massive clusters formed currently will remain bound or will disperse and contribute significantly to the stellar field population.

Aims. We measure the radial velocity dispersion of Westerlund I to explore whether the cluster is either currently in virial equilibrium, in the process of collapse, or expanding and dispersing into the field.

Methods. We obtained MIKE/Magellan high resolution optical spectra of 22 post main-sequence stars in Westerlund I for 2 or 3 epochs with a maximum baseline of about one year. Radial velocities variations between these spectra are measured by means of cross-correlation.

Results. We calculate the velocity dispersion from the cross-correlation of five yellow hypergiants and one luminous blue variable, that show little radial velocity variations between epochs and have many spectral features in common. After taking into account the effect of small number statistics and undetected binaries, we estimate the velocity dispersion for the massive stars in Westerlund I to be $2.1_{-2.1}^{+3.3} \mathrm{~km} \mathrm{~s}^{-1}$. For several different assumptions concerning possible mass segregation and the elongation of the cluster, we find that Westerlund I is subvirial at the $90 \%$ confidence level.

Conclusions. We can rule out that the cluster is significantly supervirial at the $97 \%$ confidence level, indicating that Westerlund I is currently bound. This implies that Westerlund I has survived past the point where any gas expulsion has taken place and is expected to survive for billions of years.
\end{abstract}

Key words. open clusters and associations: individual: Westerlund I - stars: kinematics and dynamics - supergiants

\section{Introduction}

Soon after its launch about twenty years ago, the Hubble Space Telescope identified many massive young star clusters in starburst and merging galaxies (Holtzman et al. 1992). These clusters are so massive that they appear unlikely to disperse in a Hubble time owing to tidal stripping (Lamers et al. 2005), which means they could possibly become globular clusters. However, violent internal or external events could cause the cluster to become unbound, in which case the cluster will dissolve into the field, contributing significantly to the stellar populations of their host galaxy. A likely process behind such an event would be the expulsion by stellar winds or the first supernovae of the remainder of the gas cloud out of which the cluster formed. This scenario has been extensively studied both analytically (Hills 1980; Elmegreen 1983; Mathieu 1983) and with $N$-body simulations (Lada et al. 1984; Goodwin \& Bastian 2006). These studies have found that the expulsion of gas lowers the potential well in which the stars reside, causing a previously virial cluster to expand. The efficacy of the gas expulsion to disrupt clusters depends on several variables, including the star formation efficiency, the timescale of the expulsion (Hills 1980; Mathieu 1983), the spatial distribution of the stars relative to the gas (Chen \& Ko 2009; Kruijssen et al. 2011), and whether the stars have achieved virial equilibrium with the potential of the surrounding gas before the gas is ejected (Goodwin 2009). Without studying the dynamical state of these clusters, we do not know which fraction of them would survive to become future globular cluster analogues and which will disperse into the field.
The only information that we have about the dynamic state of these distant massive clusters, that we can currently measure is the radial velocity dispersion (e.g. Bastian et al. 2006). Under the assumption of virial equilibrium the one-dimensional velocity dispersion can be used to estimate the mass of the cluster, if its size is resolved. This mass estimate is called the dynamical mass. The dynamical mass can then be compared to the mass derived from the luminosity of the cluster, assuming a universal IMF. Many clusters have been found to have a significantly higher velocity dispersion, than expected from the luminosity (Bastian et al. 2006). Several explanations of this have been proposed, including that: (i) the IMF might actually be different under extreme star forming conditions (Mengel et al. 2002); (ii) the velocity dispersion might be significantly higher owing to binaries (Kouwenhoven \& de Grijs 2008; Gieles et al. 2010); or (iii) the cluster might be supervirial, for example owing to a recent expulsion of gas from the cluster (Goodwin \& Bastian 2006; Baumgardt \& Kroupa 2007). These scenarios can be distinguished through observations of a cluster for which we can resolve the individual stars and thus measure the binary properties and the IMF.

Westerlund I is the most massive young cluster currently known in our Galaxy (Portegies Zwart et al. 2010). An analysis of photometric observations with the NTT/SOFI in the $J H K_{\mathrm{S}}$ bands (Brandner et al. 2008; Gennaro et al. 2011) reveals an elongated cluster with a mass of $4.9_{-0.5}^{+1.8} \times 10^{4} M_{\odot}$, a half-mass radius $r_{\mathrm{hm}}=1.0 \mathrm{pc}$, and a distance of $4 \pm 0.2 \mathrm{kpc}$. This distance was also found by comparing the radial velocity of the H II gas 
in Westerlund I with the rotation curve of the Galaxy (Kothes $\&$ Dougherty 2007). At this distance from the Galactic centre, stellar evolution and Galactic tidal stripping are expected to disperse Westerlund I over $\sim 9$ Gyr, if it is currently in virial equilibrium (Baumgardt \& Makino 2003). Thus, Westerlund I might represent a precursor of a future low-mass, metal-rich "globular cluster". However, at an age of 4.5-5 Myr (Crowther et al. 2006; Gennaro et al. 2011) we expect that Westerlund I might be out of virial equilibrium because of a recent expulsion of gas, that has not been converted into stars (Goodwin \& Bastian 2006). In this paper, we describe our measurements of the radial velocity dispersion in Westerlund I to explore whether the cluster is likely to be bound. If Westerlund I is in virial equilibrium, we expect a one-dimensional velocity dispersion of $\sim 4.6 \mathrm{~km} \mathrm{~s}^{-1}$ (see Sect. 4) for the mass and radius measured by Brandner et al. (2008) and Gennaro et al. (2011).

Mengel \& Tacconi-Garman $(2007,2009)$ previously measured the radial velocity dispersion of Westerlund I using $K$-band spectra. They found a velocity dispersion of $9.2 \pm 2.5 \mathrm{~km} \mathrm{~s}^{-1}$ based on a sample of ten stars. This value could be severely inflated owing to the presence of binaries (Kouwenhoven \& de Grijs 2008; Gieles et al. 2010). Ritchie et al. (2009a,b) also measured the radial velocities for a large sample of members and candidate members of Westerlund I using VLT/FLAMES+GIRAFFE spectra from $8350 \AA$ to $9000 \AA$ over multiple epochs to check for variability and investigate the binary properties of these massive stars.

In this paper, we present multi-epoch high-resolution spectroscopy to find the velocity dispersion corrected for binaries of Westerlund I and thus constrain the dynamical state of Westerlund I. In Sect. 2, we present the observations and discuss the data reduction. In Sect. 3, we present the radial velocity variations between epochs caused by binaries or atmospheric instabilities in the target stars, and calculate the velocity dispersion using stars of low variability. The implications of the measured velocity dispersion for the dynamical state of Westerlund I are discussed in Sect. 4. Finally we present our conclusions in Sect. 5.

\section{Observations and data reduction}

Observations were made using the MIKE spectrograph (Bernstein et al. 2003) located on the Magellan Clay telescope at the Las Campanas Observatory in June 2009, August 2009, and July 2010. MIKE has a red and a blue arm, providing two echelle spectra with a combined wavelength coverage from $3200 \AA$ to $9000 \AA$. A slit of 0.7 arcseconds was used, corresponding to a resolution of $53.000\left(\sim 6 \mathrm{~km} \mathrm{~s}^{-1}\right.$ per resolution element). Owing to the large interstellar reddening towards Westerlund I $\left(A_{\mathrm{V}}=9.6-12.3\right.$ Negueruela et al. 2010), usable spectra are only obtained redwards of $5000 \AA$. We observed 22 of the brightest spectroscopically confirmed members $(I<14.7 \mathrm{mag})$ selected from Clark et al. (2005). These stars include two of the four red supergiants, all six yellow hypergiants, a sgB[e] star, a LBV in its cool phase, and $12 \mathrm{OB}$-supergiants. All of these targets were observed for two or three epochs.

The spectra were reduced with the MIKE Redux pipeline ${ }^{1}$ with some minor adjustments. All images were bias subtracted using the overscan regions. Flat field images were derived from internal quartz lamp frames. The slit was in place during these measurements to ensure that the variation in wavelengths with

\footnotetext{
1 The MIKE Redux pipeline was written by Burles, Prochaska and Bernstein in IDL (http://web. mit. edu/ burles/wwW/).
}

the location on the CCD is similar to that in the observations. To obtain the flat field in the dark area between the orders, a diffusing glass was positioned in the optical path just downstream of the slit illuminating the area between the orders. After flat fielding we still found spurious low frequency spatial variations in the illumination of the detector above $\sim 8200 \AA$ for all spectra taken on August 2009 and July 2010, including the internal quartz lamp spectra. These features are slightly weaker for the internal quartz lamp and are spread out by the diffusing glass, suggesting that these features are created in the optical path of the telescope. To correct these variations, we calculated a smoothed flat field, which no longer contains the high frequency pixel-to-pixel variations, but only these low frequency spatial features. Although these spurious features are weaker in the flat field than in the science images, we found that they can still be roughly corrected for by dividing the flat fielded image twice by this smoothed flat field.

The curvature of the orders along the CCD is fitted using traces along the edge of the orders obtained from internal quartz lamp images. The scattered light was fitted from the dark areas between the orders by a B-spline with ten knots and this fit was then subtracted. The long slit of MIKE (five arcseconds) allowed us to identify the sky emission lines in the part of the order not illuminated by the star and subtract these emission lines. Regularly during the nights, ThAr images were taken for wavelength calibration. Two ThAr lamp images were taken at different exposure times to maximize the range of lamp line strengths that could be used in the wavelength calibration. Combined with the position of the orders on the CCD, these ThAr images were used to calculate a wavelength for every pixel on the image. The spectra were optimally extracted onto a common wavelength frame, allowing us to add the subsequent exposures of the same target. The noise was estimated at every pixel as the sum of the Poisson error in the observed flux and the readout noise. This calculated noise is consistent with the variations between multiple subsequent observations of the same target and corresponds to a $S / N$ of over 100 in the red part of the spectra. The flux was normalized using a cubic spline fit with two knots.

To correct for possible off-centre placement of the source star along the slit, we checked for zero-point shifts of the wavelength solution in every observed spectrum. To this end, we measured the shift of the telluric absorption lines in our stellar target spectra compared to a National Solar Observatory (NSO) telluric spectrum convolved to the resolution of our spectra. We selected sixteen wavelength ranges that have either no or only very weak contamination from stellar spectral lines or diffuse interstellar bands and that contain strong telluric lines. Over these wavelength ranges we calculated the peak of the cross-correlation between the NSO spectrum and the observed spectra. The shift of the zero-point of the wavelength for every spectrum is the average of the shifts calculated for the sixteen individual wavelength ranges. Figure 1 shows the difference between the individual measurements and the average offset. The distribution of these variations is quite sharply peaked $\left(\sigma=0.23 \mathrm{~km} \mathrm{~s}^{-1}\right)$. These offsets are smaller than the precision we achieved in measuring the radial velocity variations of our target stars, implying that we are not limited by the accuracy of the wavelength calibration.

\section{Results}

\subsection{Radial velocity variations}

Radial velocity variations caused by either binary stars or activity in the stellar atmospheres can inflate our measurement of 
Table 1. Observed stars.

\begin{tabular}{lcccccc}
\hline \hline id & sp. type & $13 / 14-06-2009$ & $10-08-2009$ & $09-07-2010$ & $10-07-2010$ & $\Delta v_{\max }$ \\
\hline W56 & B0 Ia & $-2.6 \pm 1.3$ & - & $0.1 \pm 0.8$ & $2.5 \pm 1.7$ & $5.1 \pm 2.9$ \\
W13 & B0.5 Ia & & spectroscopic binary & & & \\
W238 & B1 Iab & $1.2 \pm 1.5$ & - & $-1.4 \pm 0.5$ & $0.2 \pm 1.1$ & $2.6 \pm 2.0$ \\
W23a & B2 Ia & $0.6 \pm 0.8$ & - & $1.9 \pm 0.7$ & $-2.5 \pm 1.3$ & $4.4 \pm 1.9$ \\
W2a & B2 Ia & $-5.2 \pm 1.1$ & $-1.1 \pm 0.6$ & - & $6.3 \pm 1.2$ & $11.5 \pm 2.3$ \\
W11 & B2 Ia & $4.6 \pm 0.8$ & - & $-6.5 \pm 0.4$ & $1.9 \pm 0.8$ & $11.1 \pm 1.0$ \\
W28 & B2 Ia & $5.7 \pm 0.8$ & $-5.7 \pm 0.8$ & - & - & $11.3 \pm 1.7$ \\
W71 & B2.5 Ia & $-5.9 \pm 0.5$ & $5.9 \pm 0.5$ & - & - & $11.8 \pm 1.0$ \\
W70 & B3 Ia & $-6.2 \pm 0.8$ & $6.2 \pm 0.8$ & - & - & $12.3 \pm 1.5$ \\
W57a & B4 Ia & $0.4 \pm 0.2$ & $-3.3 \pm 0.4$ & $1.1 \pm 0.5$ & $1.8 \pm 0.4$ & $5.1 \pm 0.5$ \\
W33 & B5 Ia+ & $6.4 \pm 0.8$ & $1.2 \pm 1.1$ & $-7.6 \pm 0.6$ & - & $14.1 \pm 0.8$ \\
W7 & B5 Ia+ & $-4.0 \pm 0.5$ & $2.7 \pm 0.9$ & - & $1.3 \pm 0.6$ & $6.7 \pm 1.3$ \\
W16a & A5 Ia+ & $4.7 \pm 0.2$ & $-1.4 \pm 0.4$ & - & $-3.3 \pm 0.5$ & $8.0 \pm 0.6$ \\
W243 & LBV & $-0.5 \pm 0.3$ & $-0.5 \pm 0.2$ & $1.0 \pm 0.3$ & - & $1.6 \pm 0.6$ \\
W12a $^{a}$ & F1 Ia+ & $-0.7 \pm 0.4$ & $1.2 \pm 0.2$ & - & $-0.4 \pm 0.4$ & $1.9 \pm 0.5$ \\
W4 $^{a}$ & F3 Ia+ & $-2.4 \pm 0.2$ & $2.4 \pm 0.2$ & - & $-0.1 \pm 0.2$ & $4.8 \pm 0.4$ \\
W265 $^{a}$ & F1-5 Ia+ & $-1.2 \pm 0.3$ & $2.0 \pm 0.2$ & - & $-0.8 \pm 0.2$ & $3.2 \pm 0.5$ \\
W32 $^{a}$ & F5 Ia+ & $-1.8 \pm 0.1$ & $1.8 \pm 0.1$ & - & - & $3.6 \pm 0.3$ \\
W8a $^{a}$ & F8 Ia+ & $-0.8 \pm 0.1$ & $-0.4 \pm 0.1$ & $1.1 \pm 0.1$ & - & $1.9 \pm 0.2$ \\
W237 $^{2}$ & M3 Ia & $0.7 \pm 0.6$ & $-1.0 \pm 0.5$ & $0.3 \pm 1.0$ & - & $1.7 \pm 0.5$ \\
W26 $^{2}$ & M5-6 Ia & $0.6 \pm 0.2$ & $-0.2 \pm 0.1$ & $-0.5 \pm 0.3$ & - & $1.1 \pm 0.5$ \\
W9 & sgB[e] & $0.5 \pm 0.4$ & - & $-0.4 \pm 0.4$ & $-0.1 \pm 0.2$ & $0.9 \pm 0.7$ \\
\hline
\end{tabular}

Notes. The table includes the id of the star from Westerlund (1987), updated by Clark et al. (2005) (Col. 1), its spectral type (Negueruela et al. 2010; Clark et al. 2010) (Col. 2), the deviation in the velocities measured for a single epoch from the average velocity of that star (Cols. 3-5), and the maximum velocity difference between two epochs (Col. 6). All velocities are in $\mathrm{km} \mathrm{s}^{-1}$. The relative velocities have been plotted in Fig. 2 . (a) These stars were been included in our sample to calculate the radial velocity dispersion (Sect. 3.2). ${ }^{(b)}$ See Ritchie et al. (2010) for a discussion of the spectroscopic binary W13.

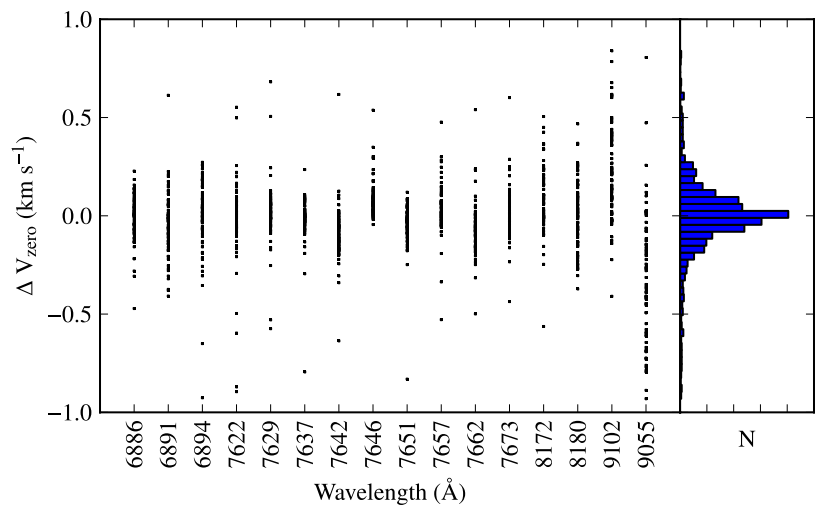

Fig. 1. Offset of the velocity zero point measured for the individual telluric lines from the averaged velocity zero point for sixteen wavelength regions containing strong telluric lines. On the $x$-axis the central wavelength of these regions is given. On the right, the histogram of all these offsets is plotted. The standard deviation in this distribution is about $230 \mathrm{~m} \mathrm{~s}^{-1}$.

the velocity dispersion of Westerlund I. To identify the variable stars, we searched for radial velocity variations between epochs over our about one year baseline. We calculated the velocity difference between epochs on a line-by-line basis, by crosscorrelating the spectra around selected spectral lines. To determine the peak of the cross-correlation on a sub-pixel level we then fit a Gaussian to the 21 points closest to the peak.

For every spectral line, the procedure described above, provided the spectral shift between any two epochs. We denote this measured spectral shift between epoch $i$ and epoch $j$ as $\Delta V_{i j}$. When we had data for more than two epochs, then the optimal estimate of the line shift between two epochs was not only measured by the direct cross-correlation between these two spectra
$\left(\Delta V_{i j}\right)$, but also through the difference in the velocities measured relative to any other epoch $k\left(\Delta V_{i k}-\Delta V_{k j}\right)$. These different determinations of the spectral shifts are not be the same in general (i.e. $\Delta V_{i j} \neq \Delta V_{i k}-\Delta V_{k j}$ ). Allende Prieto (2007) showed that it is possible to combine information about the spectral shift from both the direct cross-correlation and the spectral shifts measured relative to any other epoch, to get a more accurate estimate of the actual spectral shift, called $\Delta V_{i j}^{\prime}$ (their Eq. (3)). These estimates obey the relation $\Delta V_{i j}^{\prime}=\Delta V_{i k}^{\prime}-\Delta V_{k j}^{\prime}$, and hence can be used to assign a unique velocity to every epoch, relative to some arbitrary zero-point. We set this zero-point to the mean of the relative velocities.

Using the method from Allende Prieto (2007) we can thus estimate the relative velocities between the epochs for every spectral line. Figure 2 shows this relative velocity averaged over all spectral lines for every star, except for the spectroscopic binary W13 (Ritchie et al. 2010). The error bars are given by the standard deviation in the mean, which are an accurate representation of the uncertainty when this is dominated by random Gaussian errors as expected when the noise in the spectra is the main source of uncertainty. These velocity shifts have also been listed in Table 1, together with the largest velocity difference measured between two epochs. Only three stars (W56, W238, and W9) are consistent with having no spectral line shifts at the $2 \sigma$ level. All four of these stars tend to have large uncertainties of a few $\mathrm{km} \mathrm{s}^{-1}$. For all the other stars the radial velocity variations between the epochs are much larger than the quoted error bars, which mean that the same spectral shift is found consistently along multiple spectral lines. A similar analysis of our radial velocity standards reveals a discrepancy between our estimates and the published values (Udry et al. 1999) of about $0.4 \mathrm{~km} \mathrm{~s}^{-1}$. Although this is much smaller than the radial velocity changes that we measured in our target stars, it is still slightly larger than 




Fig. 2. Radial velocity of an epoch relative to the mean of the velocities of all epochs that the star was observed in $\mathrm{km} \mathrm{s}^{-1}$ with $1 \sigma$ uncertainties. These relative velocities have also been listed in Table 1. The vertical dotted lines delineate from left to right, the B-supergiants, the YHG hypergiants (and the LBV W243), the M supergiants, and the $\operatorname{sgB}[\mathrm{e}]$ star W9. Only the stars W56, W238 and W9 are consistent with having no radial velocity variations.

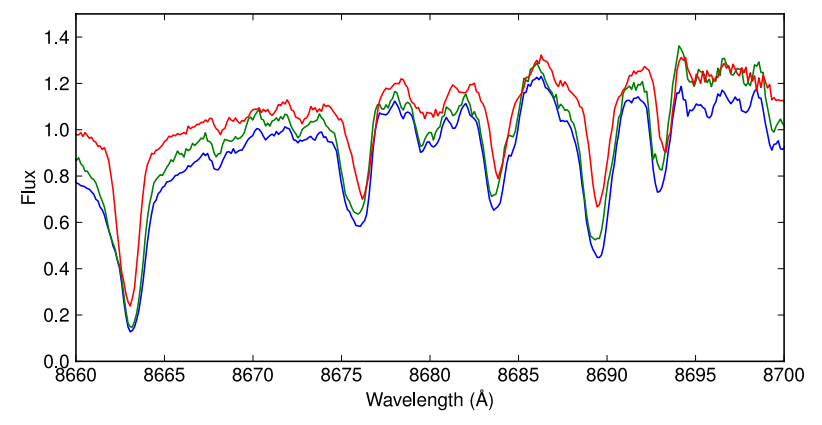

Fig. 3. Spectra of the three epochs of Wd1 237 (M3 Ia) with blue corresponding to June 2009, green to August 2009, and red to July 2010. The Ca II line at $8663 \AA$ is slightly blueshifted in July 2010 relative to the other epochs, while the other lines are clearly redshifted.

our error estimate, indicating that we might have underestimated our errors. We note that radial velocity changes in our target stars do not have to be caused by a binary star. They may instead be related to stellar activity, such as large-scale super-granulation or changes in the stellar winds. That atmospheric activity can lead to radial velocity variations of many $\mathrm{km} \mathrm{s}^{-1}$ for these massive post-main sequence stars has been well-established (e.g. de Jager 1998; Lobel et al. 1998).

That these spectral line shifts might have an atmospheric as well as a binary origin, is illustrated by the evolution of the spectrum of the M3 supergiant W237 shown in Fig. 3. Over time, the Ca II line at $8663 \AA$ is seen to evolve differently from most other lines. While the Ca II line was blueshifted in the last epoch (red line in Fig. 3) relative to the earlier observations, the other lines are clearly redshifted. These inconsistent line shifts occur repeatedly throughout the spectrum and are the cause of the relatively large errors in the relative velocity measurements of W237 relative to the other red supergiant W26 and the yellow hypergiants in Fig. 2.

Another argument for the importance of stellar activity can be made for W11, W23a, and to a lesser extent W56. These stars show a significant shift in their spectral lines over a one day baseline in July 2010. Although this could be explained by a shortperiod binary, such short-period binaries are expected to have a velocity amplitude of about $100 \mathrm{~km} \mathrm{~s}^{-1}$. Such a large velocity shift is not found relative to the other epoch of these stars, which were observed on June 2010, which could be a coincidence or caused by either a large inclination or a low mass companion. It is unlikely that these scenarios can explain the observations for all stars, suggesting that the spectral line shifts are likely to be caused by atmospheric variability. For the other stars, distinguishing between the spectral line shifts caused by binaries or stellar activity, is difficult. However, given the large observed binary fraction among massive stars, we do expect a significant portion of the radial velocity variations to be caused by binary companions (Sana et al. 2011).

The stars in Table 1 and along the $x$-axis of Fig. 2 have been roughly sorted by spectral type. The LBV is currently in a cool phase and has many lines in common with the A/F hypergiants. Figure 2 displays two trends with spectral type. The first trend is a decrease in the error bars towards later spectral types, which is caused by the far larger sample of spectral lines (most of which are less broad) available for the later type stars. The main exception on this trend is W237, which has been discussed above (see Fig. 3). The other trend is that the yellow hypergiants and the red supergiants tend to have smaller radial velocity variations than the B-supergiants. This variation is much larger than the error bars, which means that this trend is found consistently for many different spectral lines. This might be because we are sensitive to relatively close binaries with periods of at most a few years. Eldridge et al. (2008) showed that yellow hypergiants and red supergiants in close binaries evolve more quickly into a Wolf-Rayett star, because they lose their hydrogen atmosphere more effectively in binary-induced mass loss. Hence yellow hypergiants and red supergiants in close binaries spend less time in these extended phases before becoming a Wolf-Rayett star than when these stars were in wide binaries or in isolation (Clark et al. 2011). This creates a selection effect, where observed yellow hypergiants or red supergiants tend to have a small fraction of close binaries.

\subsection{Velocity dispersion}

We calculate the velocity dispersion of the cluster using only the yellow hypergiants and the LBV. This sample was chosen because these stars in general have small radial velocity variations relative to most other stars and they have a large number of spectral lines in common with each other. The large number of lines in common means that we can measure the velocity dispersion based on the cross-correlation without having to deal with the systematics, which complicate the measurement of the absolute radial velocity. When trying to include more low variability stars in this sample, we find that the extra noise caused by having to place the radial velocities on an absolute scale offsets the advantage that we gain by having a larger sample size. The choice to base our measurement of the velocity dispersion on the crosscorrelation implies that a direct comparison with previous radial velocity measurements of the stars in Westerlund I is impossible (Mengel \& Tacconi-Garman 2007, 2009; Ritchie et al. 2009a,b). 


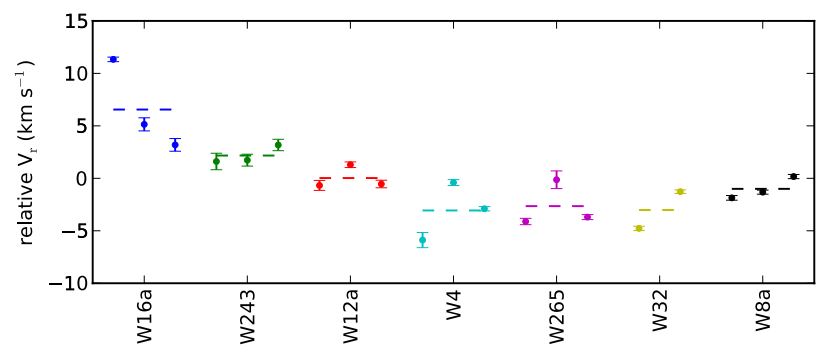

Fig. 4. Velocities of all epochs of the YHG and the LBV relative to each other. The horizontal dashed lines show the mean velocity for every star. The zero-point of the $y$-axis has been arbitrarily set to the mean of all the velocities.

Using the same method as in our search for radial velocity variations, we used cross-correlations to calculate the relative velocities between the different stars in our sample. The relative velocities for all the epochs of the yellow hypergiants is shown in Fig. 4 with the mean relative velocity for every star marked as a dashed line. We can see that the velocity variations between epochs of the same star is of the same order as the velocity differences between stars. This implies that Westerlund I has a very small intrinsic velocity dispersion. On the basis of the outlying average velocity and the large velocity change between epochs, we identified W16a as a binary star (or as having a very active atmosphere) and excluded it from our calculation of the velocity dispersion. Below we calculate the velocity dispersion based on the other six stars.

Owing to the small number of stars in our sample, we have a large statistical uncertainty in the measured velocity dispersion. To calculate this statistical uncertainty, we evaluated the likelihood that the observed distribution would have been drawn from a cluster with a Gaussian radial velocity distribution of standard deviation $\sigma_{\mathrm{v}}$ and mean $\mu$

$P\left(\sigma_{\mathrm{v}}, \mu\right)=\prod_{i} \frac{1}{\sqrt{2 \pi\left(\sigma_{\mathrm{v}}^{2}+\sigma_{i}^{2}\right)}} \exp \left(-\frac{\left(v_{i}-\mu\right)^{2}}{2\left(\sigma_{\mathrm{v}}^{2}+\sigma_{i}^{2}\right)}\right)$,

where $v_{i}$ is the observed mean velocity of the star and $\sigma_{i}$ is the uncertainty in the measured velocity. The likelihood that the observations were drawn from a distribution with a given velocity dispersion is calculated by integrating over $\mu$. With only twothree epochs over a one year baseline and most, if not all, stars being variable the uncertainties on the measured radial velocities of the stars $\left(\sigma_{i}\right)$ are not well-determined. Owing to this, we considered two cases. In the first case, we set $\sigma_{i}^{2}$ to the variance of the velocity spread between epochs. This leads to the probability distribution shown as the blue dashed line in Fig. 5 and corresponds to an intrinsic velocity dispersion of $\sigma=1.7_{-1.7}^{+3.1} \mathrm{~km} \mathrm{~s}^{-1}$, where the errors give the $95 \%$ confidence limits. Alternatively, we set $\sigma_{i}$ to zero, leading to a velocity dispersion of $\sigma=2.1_{-0.9}^{+3.4} \mathrm{~km} \mathrm{~s}^{-1}$. This case is shown as the red dotted line in Fig. 5. We note that the peak in the second case overlaps exactly with the standard deviation in the measured stellar velocities $\left(2.14 \mathrm{~km} \mathrm{~s}^{-1}\right)$.

The second case, with $\sigma_{i}=0$, can be seen as an "upperlimit estimate" of the probability distribution of the true velocity dispersion of Westerlund I. This velocity dispersion would be accurate, if the errors were much smaller than $1 \mathrm{~km} \mathrm{~s}^{-1}$. Given the large radial velocity variations measured between epochs, as well as the possibility of undetected binaries with a period of longer than five years and a velocity amplitude of tens of $\mathrm{km} \mathrm{s}^{-1}$, it is very unlikely that the uncertainties in our estimate of the

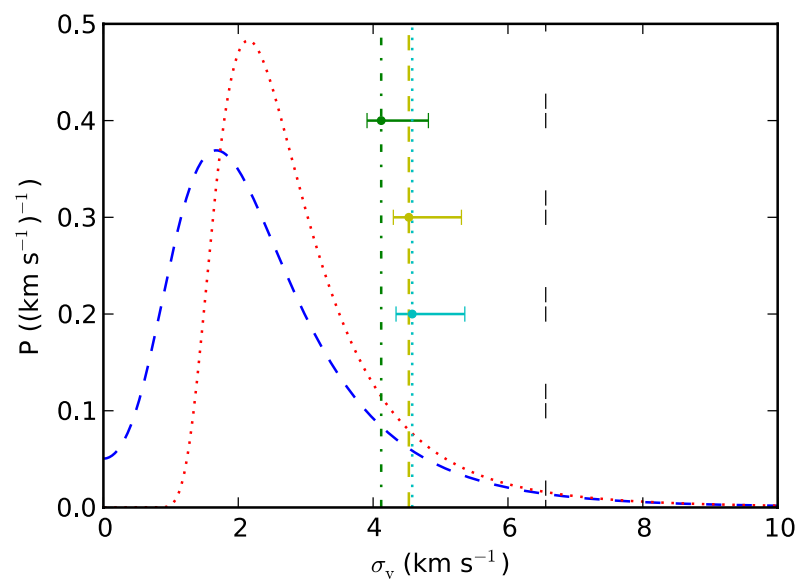

Fig. 5. Our estimate of the probability distribution of the velocity dispersion for the massive stars in Westerlund I. The blue dashed line shows the case where the measurement error is set by the variation in the radial velocity between epochs. The red dotted line shows the limit, where we assume no measurement errors. The vertical lines mark the velocity dispersion expected in virial equilibrium under several assumptions explained in Sect. 4. From the highest to lowest estimates, we have the velocity dispersion calculated for a spherical, non-mass-segregated cluster for the mass estimates from Negueruela et al. (2010) (black, long dashed) and Gennaro et al. (2011) (cyan, dotted). For the mass estimate of Gennaro et al. (2011) we also show the expected velocity dispersion taking into account the velocity anisotropy, which is consistent with the elongation of Westerlund I (yellow, dashed), and the case where we take into account both the velocity anisotropy and the possible mass segregation (green, dash-dot). The error bars represent the quoted uncertainty in the photometric mass of Gennaro et al. (2011).

systematic radial velocity are smaller than $1 \mathrm{~km} \mathrm{~s}^{-1}$. However, including more accurate measurement errors would only decrease the measured velocity dispersion, as illustrated by the case where we set the measurement errors to the variance of the velocity variations between epochs (blue dashed line in Fig. 5). Increasing $\sigma_{i}$ does not change the probability at the larger velocity dispersions significantly, i.e. the upper bound of the $95 \%$ confidence limit remains around $5 \mathrm{~km} \mathrm{~s}^{-1}$. Since the uncertainties are not well-defined, we conservatively focus on the case with $\sigma_{i}=0$.

\section{Discussion}

To explore the virial state of the cluster, we compared our measurement of the velocity dispersion with the velocity dispersion expected in virial equilibrium for the photometric mass of Westerlund I. We calculated a first estimate of the velocity dispersion expected in virial equilibrium assuming that Westerlund I is not mass segregated and that the velocities are isotropic. In virial equilibrium, the photometric mass is equal to the dynamical mass, which under these assumptions is given by:

$M_{\mathrm{dyn}}=\eta \frac{\sigma_{\mathrm{rad}}^{2} r_{\mathrm{hm}}}{G}$

where $\eta \approx 10$ is appropriate for the density profile found by Brandner et al. (2008), $\sigma_{\text {rad }}$ is the radial velocity dispersion, and $r_{\mathrm{hm}} \approx 1.0 \mathrm{pc}$ (Brandner et al. 2008) is the half mass radius. On the basis of an extrapolation from the number of massive stars in Westerlund I, Negueruela et al. (2010) estimated a stellar mass of about $10^{5} M_{\odot}$. In virial equilibrium, this corresponds to a velocity dispersion of $\sim 6.5 \mathrm{~km} \mathrm{~s}^{-1}$. This is far larger than 
our measured velocity dispersion, implying that Westerlund I is subvirial at the $98 \%$ confidence level. An alternative photometric mass estimate was given by Gennaro et al. (2011). On the basis of an extrapolation of the measured IMF for the intermediate mass stars between $3.5 M_{\odot}$ and $27 M_{\odot}$, these authors found a lower stellar mass of $4.9_{-0.5}^{+1.8} \times 10^{4} M_{\odot}$. In virial equilibrium, we would then expect a velocity dispersion of $4.6_{-0.3}^{+0.8} \mathrm{~km} \mathrm{~s}^{-1}$. For this velocity dispersion, our measurements are consistent with a subvirial cluster at the $91 \%$ confidence level.

In the conversions from the photometric mass to the velocity dispersion calculated above, we have assumed that the cluster has an isotropic velocity distribution and is not masssegregated. Both of these assumptions are unlikely to be valid for Westerlund I. An isotropic velocity distribution would generally lead to a spherical cluster. However, Westerlund I is observed to be elongated, with surfaces of constant density lying on ellipses rather than circles (Gennaro et al. 2011). The exact implications of this elongation on the expected velocity dispersion depend on its origins. Under the assumption that this elongation is supported by a velocity anisotropy and thus is not a transient on the dynamical timescale, we calculated in Appendix A the expected velocity dispersion. We found that the dynamical mass is given by Eq. (A.7), which is reproduced as

$M_{\text {dyn }}=\eta_{\text {ellips }} \eta \frac{\sigma_{\mathrm{rad}}^{2} r_{\mathrm{hm}}^{\prime}}{G}$,

where $r_{\mathrm{hm}}^{\prime}$ is the half mass radius, assuming that the density profile in all dimensions is the same as along the observed semimajor axis. $r_{\mathrm{hm}}^{\prime}$ can be estimated from the half-mass radius of $1.0 \mathrm{pc}$ (Brandner et al. 2008) and the ellipticity of Westerlund I of 0.75 (Gennaro et al. 2011) to be roughly $1.2 \mathrm{pc}$. The correction factor for the ellipticity of the cluster $\eta_{\text {ellips }}$ is approximately 0.85 , if the axis along the line of sight is not much shorter than the observed axes. The other parameters in the equation are the same as in Eq. (2). For the photometric mass estimate of $4.9_{-0.5}^{+1.8} \times 10^{4} M_{\odot}($ Gennaro et al. 2011), taking into account the elongation leads to a only slightly smaller expected velocity dispersion of $4.5_{-0.2}^{+0.8} \mathrm{~km} \mathrm{~s}^{-1}$.

Owing to the tendency of massive stars to reside at the center of massive clusters (i.e. mass segregation), the velocity dispersion of the massive stars that we measured might not be representative of the cluster as a whole (Fleck et al. 2006). To calculate the effect of mass segregation on the difference between the measured and expected velocity dispersion, we separate the cluster into two populations. The first population represents the massive post-main sequence stars from which our sample was drawn, with a half-mass radius of $0.44 \mathrm{pc}$ and a mass of $6 \times 10^{3} M_{\odot}$ (Clark et al. 2005; Negueruela et al. 2010). The second population contains the remaining low and intermediate mass stars in the cluster, with a half-mass radius of $1.0 \mathrm{pc}$ and a much higher total mass of $4.3 \times 10^{4} M_{\odot}$ (Brandner et al. 2008). For a cluster whose stellar volume density is well-represented by a Plummer sphere, we found that in virial equilibrium the velocity dispersion of the total population is about $10 \%$ larger than for the subset of massive stars using a two-fluid approximation (Eq. (7.158) in Binney \& Tremaine 2008). This would lead us to expect to measure a velocity dispersion of roughly $4.1_{-0.2}^{+0.7} \mathrm{~km} \mathrm{~s}^{-1}$ for a mass-segregated, elongated cluster.

Besides showing the probability distribution of the velocity dispersion of the massive stars in Westerlund I implied by our observations, Fig. 5 also illustrates the velocity dispersions expected in virial equilibrium based on the various assumptions discussed above. Even for the lowest estimate, our observations still imply that Westerlund I is either subvirial or virial. For the probability distribution including a rough estimate of our measurement errors, we inferred a $91 \%$ probability of the observed velocity dispersion being smaller than the smallest estimate of $4.1 \mathrm{~km} \mathrm{~s}^{-1}$. For the more conservative probability distribution, which assumes no measurement errors, we found a $87 \%$ probability.

For the cluster to be unbound, the kinetic energy has to be higher than the binding energy, which would correspond to a velocity dispersion $\sqrt{2}$ larger than expected in virial equilibrium. This can be excluded with at least $97 \%$ confidence from our measurements, with the exact percentage depending on the assumptions made. This implies that Westerlund I is bound and has survived any recent gas expulsion. To survive the gas expulsion, Westerlund I should either have had a high star-formation efficiency, which would cause the cluster to remain close to virial equilibrium or the stars in the cluster should have been dynamically cold at the moment the gas was expelled (Goodwin 2009). If the case of Westerlund I were typical of a massive cluster, the excessive velocity dispersion measured for extragalactic clusters would not automatically be indicative of a supervirial cluster, but have another origin, for example in binaries (Gieles et al. 2010). In any case, Westerlund I itself will not disperse into the field quickly and likely persist for billions of years.

\section{Conclusions}

We have presented multi-epoch high resolution spectra of 22 massive post main-sequence stars in the young, massive, Galactic cluster Westerlund I. For 21 of these 22 stars, the velocity variations have been measured between multiple epochs by means of cross-correlation (see Table 1 or Fig. 2). Many of these stars seem to show radial velocity variations at the one to ten $\mathrm{km} \mathrm{s}^{-1}$ level. Some of these variations might be explained by an underestimate of our uncertainties. Alternatively, these variations could be caused by either binaries or instabilities/waves in the complex atmospheres of these super- and hypergiants.

Using the five least variable yellow hypergiants and the LBV, we have measured a velocity dispersion for the massive stars in Westerlund I of $\sigma=2.1_{-0.9}^{+3.4} \mathrm{~km} \mathrm{~s}^{-1}$ with a confidence limit of $95 \%$, assuming no significant errors in the measured radial velocity differences between the stars. In reality, even these least variable stars have displayed radial velocity variations between epochs of a few $\mathrm{km} \mathrm{s}^{-1}$, implying that the quoted velocity dispersion is only an upper limit to the true velocity dispersion.

In Fig. 5, the probability distribution of the velocity dispersion from our measurements have been compared to several estimates of the velocity dispersion that we expect in virial equilibrium. These estimates have been calculated using different estimates of the total mass of Westerlund I, as well as different rough approximations of the effects of both possible mass segregation and a velocity anisotropy, as suggested by the observed spatial elongation of Westerlund I. For all of these cases the observations are consistent with those of a subvirial cluster. For the lower photometric mass estimate of Gennaro et al. (2011), the observations are also consistent with the cluster in virial equilibrium. In all cases, we have excluded that Westerlund I is significantly supervirial, implying that the cluster is bound, and that it has survived any recent gas expulsion. Barring any violent interactions with other clusters or molecular clouds, Westerlund I, which might be the most massive young cluster in our Galaxy, is expected to survive for billions of years. 
Acknowledgements. NSO/Kitt Peak FTS data used here were produced by NSF/NOAO. We would like to thank Justin Read, Kevin Covey, Richard Parker, Phil Massey, and Hans Martin Schmidt for helpful discussions. We are also very grateful to an anonymous referee, whose comments and suggestions led to major improvements in the paper.

\section{Appendix A: Velocity dispersion in an ellipsoidal cluster}

The density distribution of both the massive and intermediate mass stars in Westerlund I has been shown to be elongated (Negueruela et al. 2010; Gennaro et al. 2011). We calculated the effect of this elongation on the dynamical mass calculation of Westerlund I, assuming an ellipsoidal density profile, as suggested by Gennaro et al. (2011), who fitted a density profile to their stellar density distribution of Westerlund I, where the lines of constant densities lie along ellipses instead of circles. To limit the number of free parameters as well as greatly simplify the equations, we assumed that one of the axis of the ellipsoid lies along the line of sight. The other two axes are then the axes found by Gennaro et al. (2011) in the plane of the sky. We defined the length of the semi-major axis found by Gennaro et al. (2011) as $a_{1}$, the length of the semi-minor axis as $a_{2}$, and the length along the line of sight as $a_{3}$, the last of which is unconstrained by observations.

For an ellipsoidal cluster in virial equilibrium, an elongation implies a velocity anisotropy, which is given by the tensor form of the virial theorem

$2 K_{j j}+W_{j j}=0$

where $2 K_{j j}=M_{\mathrm{dyn}} \sigma_{j}^{2}$ is the tensor form of the kinetic energy along the axis $j$ and $W_{j j}$ is the potential energy tensor. For an ellipsoidal system, this potential can be represented as the in a product of two factors (see Eq. (2.144) in Binney \& Tremaine 2008), one that depends only on the axial ratios of the cluster, and another that is independent of either the clusters ellipticity or the axis along which the potential energy tensor is calculated and depends only on the density dropoff along a single axis, which we take to be the observed semi-major axis. In functional form, this corresponds to

$W_{j j}=-f_{j}\left(a_{1}, a_{2}, a_{3}\right) * g\left(\rho_{\text {along semi-major axis }}\right)$,

with

$f_{j}\left(a_{1}, a_{2}, a_{3}\right)=\frac{3}{2} \frac{a_{2} a_{3}}{a_{1}^{2}}\left(\frac{a_{j}}{a_{1}}\right)^{2} A_{j}$,

where the $A_{j}$ are a complex function of the axial ratios given by Table 2.1 in Binney \& Tremaine (2008). We note that the equations in this table assume that $a_{1}>a_{2}>a_{3}$, which will make it necessary to rearrange the indices, if the axis along the line of sight is not the shortest.

Since $g$ does not depend on both $a_{2}$ and $a_{3}$, we can calculate this term for the spherical case, where we set $a_{2}$ and $a_{3}$ to $a_{1}$. For a spherical cluster we have $A_{j}=2 / 3$, so $f_{j}\left(a_{1}, a_{2}, a_{3}\right)=1$ and $W_{j j}=\frac{1}{3} W$, where $W$ is the total potential energy. Using the equation for the dynamical mass of a spherical cluster (e.g. Portegies Zwart et al. 2010), we get

$W_{j j, \mathrm{circ}}=-g=-\frac{1}{\eta} \frac{G M_{\mathrm{dyn}}^{\prime 2}}{r_{\mathrm{hm}}^{\prime}}$,

where $\eta \approx 10$ (Portegies Zwart et al. 2010) for the density profiles fitted by Brandner et al. (2008) and Gennaro et al. (2011),

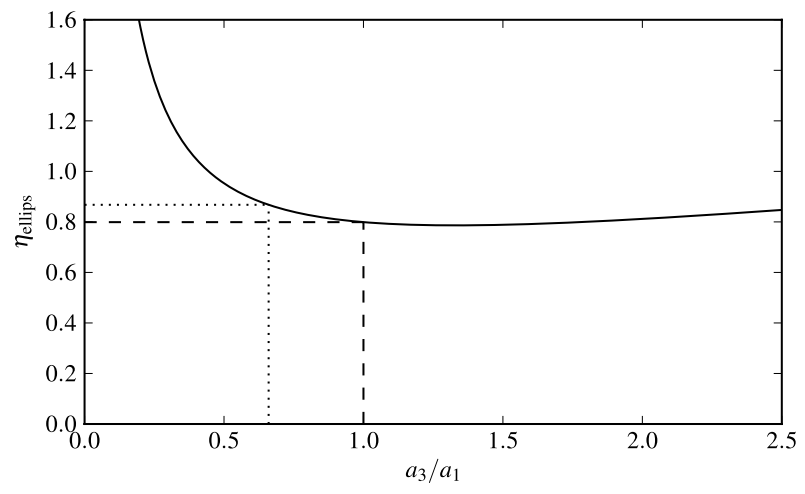

Fig. A.1. Correction factor $\eta_{\text {ellips }}$ needed to take into account the spheroidal shape of the cluster plotted against the ratio of the length of the axis along the line of sight to the length of the observed semi-major axis for an observed ellipticity of $0.75\left(a_{2} / a_{1} \approx 0.66\right)$. The cases where the density dropoff along the line of sight is the same as the semi-major axis (dashed) and the same as the minor axis (dotted) are shown.

$M_{\mathrm{dyn}}^{\prime}$ is the mass of this circular cluster, and $r_{\mathrm{hm}}^{\prime}$ is the half-mass radius of this cluster. The dynamical mass of this artificial spherical cluster is related to the mass of the spheroidal cluster with

$\frac{M_{\mathrm{dyn}}^{\prime}}{M_{\mathrm{dyn}}}=\frac{a_{1}^{3}}{a_{1} a_{2} a_{3}}$.

Entering Eqs. (A.3)-(A.5) into the equation for the ellipsoidal potential energy tensor gives

$W_{j j}=-\frac{3}{2} \frac{a_{j}^{2}}{a_{2} a_{3}} A_{j} \frac{1}{\eta} \frac{G M_{\mathrm{dyn}}^{2}}{r_{\mathrm{hm}}^{\prime}}$.

Combined with the virial equation (Eq. (A.1)) for the radial velocity dispersion $(j=3)$ this can be converted as

$M_{\mathrm{dyn}}=\eta_{\mathrm{ellips}} \eta \frac{\sigma_{\mathrm{rad}}^{2} r_{\mathrm{hm}}^{\prime}}{G}$,

where the correction for the ellipsoidal structure has been completely integrated in $\eta_{\text {ellips }}$, which is defined as

$\eta_{\text {ellips }} \equiv \frac{2}{3} \frac{a_{2}}{a_{3}} \frac{1}{A_{3}}$.

This correction factor depends only on the axial ratios. For an ellipticity along the line of sight of $0.75\left(a_{2} / a_{1} \approx 0.66\right)$ (Gennaro et al. 2011), we plot the dependence of this correction factor on $a_{3} / a_{1}$ in Fig. A.1. This figure shows that there is only a small dependence of the correction factor on the actual length of the axis along the line of sight for $a_{3} / a_{1}>0.5$. In this range, an increase in the length of the axis causes the total potential and thus the total kinetic energy to drop. However, this sharp decline in kinetic energy mostly corresponds to a lowering of the velocity dispersion in the plane of the sky, with the radial velocity dispersion staying roughly constant. This interplay between the change in the total kinetic energy and the division of this kinetic energy over the three axes produces the curve shown in Fig. A.1.

\section{References}

Allende Prieto, C. 2007, AJ, 134, 1843

Bastian, N., Saglia, R. P., Goudfrooij, P., et al. 2006, A\&A, 448, 881 Baumgardt, H., \& Kroupa, P. 2007, MNRAS, 380, 1589 
Baumgardt, H., \& Makino, J. 2003, MNRAS, 340, 227

Bernstein, R., Shectman, S. A., Gunnels, S. M., Mochnacki, S., \& Athey, A. E.

2003, in SPIE Conf. 4841, ed. M. Iye, \& A. F. M. Moorwood, 1694

Binney, J., \& Tremaine, S. 2008, Galactic Dynamics, ed. J. Binney, \& S.

Tremaine, second edition (Princeton University Press)

Brandner, W., Clark, J. S., Stolte, A., et al. 2008, A\&A, 478, 137

Chen, H.-C., \& Ko, C.-M. 2009, ApJ, 698, 1659

Clark, J. S., Negueruela, I., Crowther, P. A., \& Goodwin, S. P. 2005, A\&A, 434, 949

Clark, J. S., Ritchie, B. W., \& Negueruela, I. 2010, A\&A, 514, A87

Clark, J. S., Ritchie, B. W., Negueruela, I., et al. 2011, A\&A, 531, A28

Crowther, P. A., Hadfield, L. J., Clark, J. S., Negueruela, I., \& Vacca, W. D. 2006, MNRAS, 372, 1407

de Jager, C. 1998, A\&ARv, 8, 145

Eldridge, J. J., Izzard, R. G., \& Tout, C. A. 2008, MNRAS, 384, 1109

Elmegreen, B. G. 1983, MNRAS, 203, 1011

Fleck, J., Boily, C. M., Lançon, A., \& Deiters, S. 2006, MNRAS, 369, 1392

Gennaro, M., Brandner, W., Stolte, A., \& Henning, T. 2011, MNRAS, 412, 2469

Gieles, M., Sana, H., \& Portegies Zwart, S. F. 2010, MNRAS, 402, 1750

Goodwin, S. P. 2009, Ap\&SS, 324, 259

Goodwin, S. P., \& Bastian, N. 2006, MNRAS, 373, 752

Hills, J. G. 1980, ApJ, 235, 986
Holtzman, J. A., Faber, S. M., Shaya, E. J., et al. 1992, AJ, 103, 691

Kothes, R., \& Dougherty, S. M. 2007, A\&A, 468, 993

Kouwenhoven, M. B. N., \& de Grijs, R. 2008, A\&A, 480, 103

Kruijssen, J. M. D., Maschberger, T., Moeckel, N., et al. 2011, MNRAS, 1779

Lada, C. J., Margulis, M., \& Dearborn, D. 1984, ApJ, 285, 141

Lamers, H. J. G. L. M., Gieles, M., Bastian, N., et al. 2005, A\&A, 441, 117

Lobel, A., Israelian, G., de Jager, C., et al. 1998, A\&A, 330, 659

Mathieu, R. D. 1983, ApJ, 267, L97

Mengel, S., \& Tacconi-Garman, L. E. 2007, A\&A, 466, 151

Mengel, S., \& Tacconi-Garman, L. E. 2009, Ap\&SS, 324, 321

Mengel, S., Lehnert, M. D., Thatte, N., \& Genzel, R. 2002, A\&A, 383, 137

Negueruela, I., Clark, J. S., \& Ritchie, B. W. 2010, A\&A, 516, A78

Portegies Zwart, S. F., McMillan, S. L. W., \& Gieles, M. 2010, ARA\&A, 48, 431

Ritchie, B. W., Clark, J. S., Negueruela, I., \& Crowther, P. A. 2009a, A\&A, 507, 1585

Ritchie, B. W., Clark, J. S., Negueruela, I., \& Najarro, F. 2009b, A\&A, 507, 1597

Ritchie, B. W., Clark, J. S., Negueruela, I., \& Langer, N. 2010, A\&A, 520, A48

Sana, H., James, G., \& Gosset, E. 2011, MNRAS, 416, 817

Udry, S., Mayor, M., Maurice, E., et al. 1999, in Precise Stellar Radial Velocities, ed. J. B. Hearnshaw, \& C. D. Scarfe, ASP Conf. Ser., 185, IAU Colloq., 170,383

Westerlund, B. E. 1987, A\&AS, 70, 311 